ISSN 2306-1561

Automation and Control in Technical Systems (ACTS)

2014, No 1.2(9), pp. 83-92.

DOI: $10.12731 / 2306-1561-2014-1-22$

\title{
Hardware Setup for priority service traffic corporate network
}

\section{Skvortsova Tatiana Ivanovna}

Russian Federation, Ph. D., Associate Professor, Department of «Automated Control Systems».

Moscow State University Instrument Engineering and Computer Science (MGUPI), 107996, Russian Federation, Moscow, Str. Stromynka, 20. Tel.: +7 (499)268-00-01, http://www.mgupi.ru

\section{skv-tata@yandex.ru}

\begin{abstract}
This article describes the principles of prioritizing traffic for packets transmitted over the network by assigning a specific class of service and provide for them the appropriate quality of service. They provide stable operation of modern multimedia applications. Traffic Shaping is solved by technology QoS. For specific router (Cisco 2911) defined command line control QoS for prioritizing multimedia information.
\end{abstract}

Keywords: methods of service quality, QoS, service class, traffic prioritization, service models QoS, classification and marking of packages. 
ISSN 2306-1561

Автоматизация и управление в технических системах (АУТС) 2014. - №1.2(9). - C. 83-92.

DOI: $10.12731 / 2306-1561-2014-1-22$

\section{УДК 004.9}

\section{Настройка оборудования для приоритетного обслуживания трафика корпоративной сети}

\section{Скворцова Татьяна Ивановна}

Российская Федерация, кандидат технических наук, доцент кафедры «Автоматизированные системы управления и информационные технологии».

ФГБОУ ВПО «Московский государственный университет приборостроения и информатики (МГУПИ)», 107996, Российская Федерация, г. Москва, ул. Стромынка, д. 20, Тел.: +7 (499)26800-01, http://www.mgupi.ru

\section{skv-tata@yandex.ru}

Аннотация. $\mathrm{B}$ статье описываются принципы приоритезации трафика для передаваемых по сети пакетов путем присвоения им определенного класса обслуживания и обеспечения для них соответствующего качества обслуживания. Они обеспечивают устойчивую работу современных мультимедийных приложений. Приоритезация трафика решается с помощью технологии QoS. Для конкретного маршрутизатора (Cisco 2911) определена командная строка управления QoS для приоритезации мультимедийной информации.

Ключевые слова: методы качества обслуживания, QoS, класс обслуживания, приоритезация трафика, сервисные модели QoS, классификация и разметка пакетов.

\section{1. Введение}

Методы обеспечения качества обслуживания - QoS (Quality of Service) занимают сегодня важное место в арсенале технологий корпоративных сетей. Они обеспечивают устойчивую работу современных мультимедийных приложений, таких как IP телефония, видео- и радиовещание, интерактивное дистанционное обучение и т.д. Методы QoS направлены на улучшение характеристик производительности и надежности сети и позволяют уменьшить задержки, потери пакетов в периоды перегрузки сети $[1,2,7]$.

Построить корпоративную сеть, которая гарантированно пропускала бы весь трафик в случае активной сетевой работы всех пользователей, практически нереально. Параметры пропускной способности рассчитываются по неким усредненным 
показателям с учетом предположений о характере использования сети (типы задач, наличие голосового и мультимедийного трафика).

В большинстве корпоративных сетей малых и средних предприятий пропускная способность сети используется менее чем на $10 \%$, и ограничения в передаче данных изза исчерпания полосы пропускания кажутся маловероятными. Но все каналы связи имеют свои пределы. С увеличением интенсивности использования сетевых приложений вероятность кратковременной перегрузки сети будет только повышаться $[7-15]$.

Сама сеть не гарантирует доставку информации. Если пакет с данными не может быть передан, он удаляется и, естественно, теряется. Большинство приложений корректно обработает факты потери части передаваемых данных и запросит их повторно. Однако есть задачи, для которых любая потеря пакетов недопустима. Например, при передаче голоса или видео данных подобная ситуация приведет к возникновению "провалов" [8, 10, 13]. В этом случае можно решить проблему, если предоставить передаче голоса и видео более привилегированные условия, чем, например, протоколу пересылки почтовых сообщений.

\section{2. Приоритезация трафика}

Задача приоритезации трафика решается путем присвоения передаваемым по сети пакетам определенного класса обслуживания и обеспечения для них соответствующего качества обслуживания. Часто для простоты все эти технологии называют QoS. Данная задача является весьма сложной и решается по-разному для локальных и магистральных сетей [3].

QoS - способность сети обеспечить необходимый сервис заданному трафику в определенных технологических рамках.

Необходимый сервис описывается многими параметрами, отметим среди них самые важные.

Bandwidth (BW) - полоса пропускания, описывает номинальную пропускную способность среды передачи информации, определяет ширину канала. Измеряется в bit/s (bps), kbit/s (kbps), mbit/s (mbps).

Delay - задержка при передаче пакета.

Jitter - колебание (вариация) задержки при передаче пакетов.

Packet Loss - потери пакетов. Определяет количество пакетов, отбрасываемых сетью во время передачи.

Чаще всего для описания пропускной способности канала проводят аналогию с водопроводной трубой. В еe рамках Bandwidth - это ширина трубы, a Delay - длина (рисунок 1). 


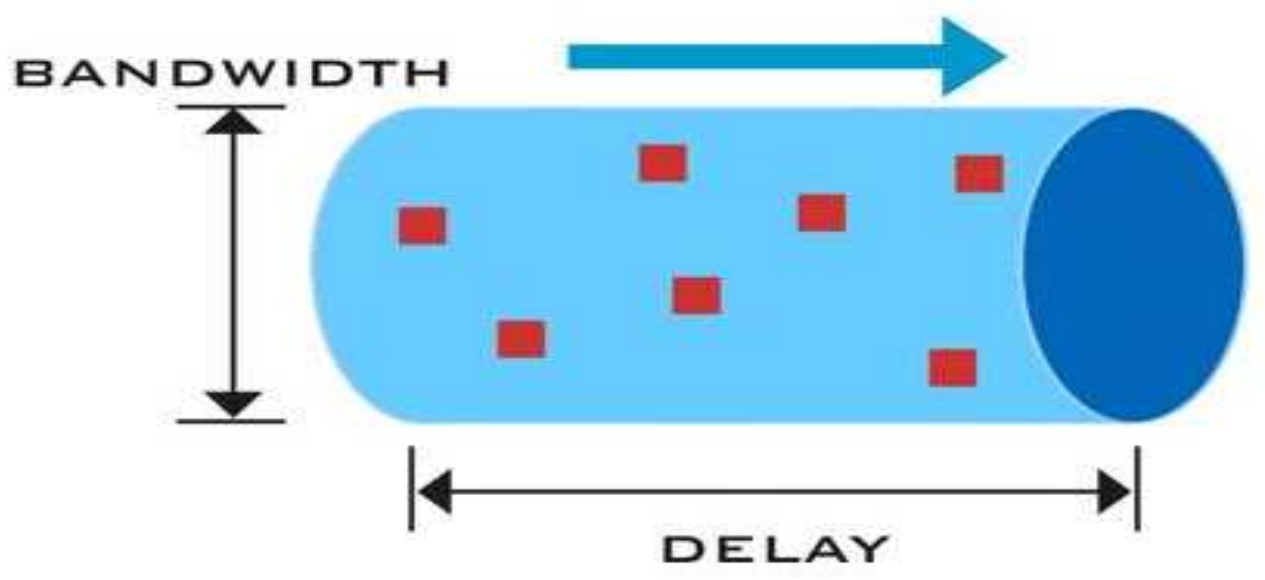

Рисунок 1 - Пропускная способность

\section{3. Сервисные модели QoS}

Best Effort Service

Негарантированная доставка. Абсолютное отсутствие механизмов QoS. Используются все доступные ресурсы сети без какого-либо выделения отдельных классов трафика и регулирования. Считается, что лучшим механизмом обеспечения QoS является увеличение пропускной способности. Это в принципе правильно, однако некоторые виды трафика (например, голосовой) очень чувствительны к задержкам пакетов и вариации скорости их прохождения. Модель Best Effort Service даже при наличии больших резервов допускает возникновение перегрузок в случае резких всплесков трафика. Поэтому были разработаны и другие подходы к обеспечению QoS.

Integrated Service (IntServ)

Integrated Service - модель интегрированного обслуживания. Может обеспечить сквозное (End-to-End) качество обслуживания, гарантируя необходимую пропускную способность. IntServ использует для своих целей протокол сигнализации RSVP. Позволяет приложениям выражать сквозные требования к ресурсам и содержит механизмы обеспечения данных требований. IntServ можно кратко охарактеризовать как резервирование ресурсов (Resource reservation).

Differentiated Service (DiffServ)

Differentiated Service - модель дифференцированного обслуживания. Определяет обеспечение QoS на основе четко определенных компонентов, комбинируемых с целью предоставления требуемых услуг. Архитектура DiffServ предполагает наличие классификаторов и формирователей трафика на границе сети. Разделяет трафик на классы, вводя несколько уровней QoS. DiffServ состоит из следующих функциональных блоков: граничные формирователи трафика (классификация пакетов, маркировка, управление интенсивностью) и реализаторы политики (распределение ресурсов, политика отбрасывания пакетов). DiffServ можно кратко охарактеризовать как приоритезацию трафика (Prioritization).

Classification and Marking 
Classification and Marking - модель «Классификация и разметка». Классификация пакетов (Packet Classification) представляет собой механизм соотнесения пакета к определенному классу трафика. Другой не менее важной задачей при обработке пакетов является маркировка пакетов (Packet Marking) - назначение соответствующего приоритета (метки).

В зависимости от уровня рассмотрения (имеется в виду семиуровневая модель взаимодействия открытых систем) эти задачи решаются по-разному.

\section{4. Классификация и разметка пакетов на сетевом уровне}

Маршрутизирующее оборудование (Layer 3) оперирует IP пакетами, в которых под цели маркировки предусмотрено соответствующее поле в заголовке - IP Type of Service (ToS) размером один байт (рисунок 2). ToS может быть заполнен классификатором IP Precedence или DSCP (Differentiated Services Code Point, точка кода дифференцированных услуг) в зависимости от типа задачи [4].

DSCP - это поле в заголовке IP пакета, которое используется в целях классификации передаваемой информации.

IP Precedence (IPP) имеет размерность 3 бита (принимает значения 0 - 7). DSCP относится к модели DiffServ и состоит из 6 бит (значения 0 - 63).

Кроме цифровой формы, значения DSCP могут быть выражены с использованием специальных ключевых слов: доставка по возможности BE - Best Effort, гарантированная доставка AF - Assured Forwarding и срочная доставка EF - Expedited Forwarding. В дополнение к этим трем классам существуют коды селектора классов, которые добавляются к обозначению класса и обратно совместимы с IPP. Например, значение DSCP равное 26 можно записать как AF31, что полностью равнозначно.

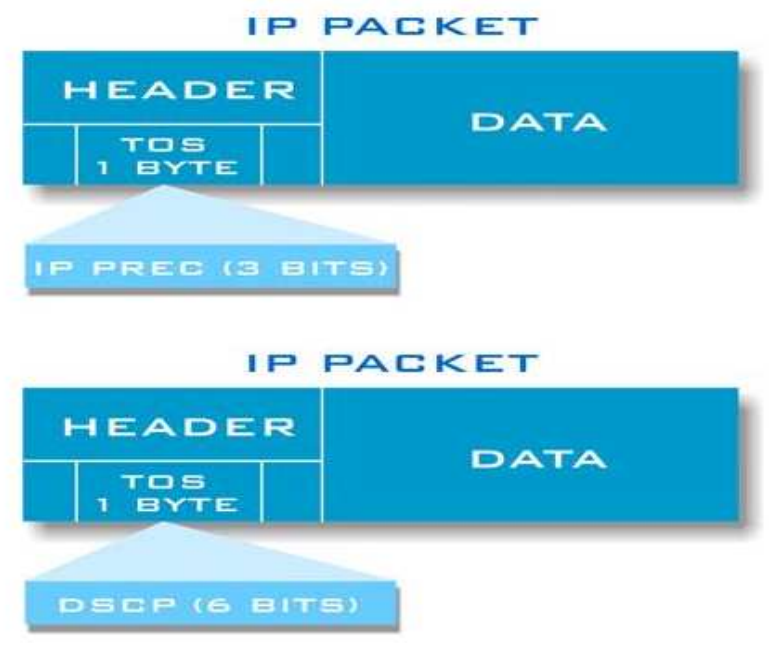

\section{Рисунок 2 - Заголовок IP пакета}

Технология MPLS (Multi Protocol Label Switching - многопротокольная коммутация по меткам) содержит индикатор QoS внутри метки в соответствующих битах MPLS EXP (3 бита). 


\section{5. Оборудование Cisco}

Cisco Systems Inc. в настоящее время является лидером мирового рынка в сегменте сетевых решений. Кроме того, разработки в сфере программного и аппаратного обеспечения активно применяются для построения локальных компьютерных сетей (ЛКС). Активное сетевое оборудование и семейство межсетевых платформ гарантируют максимально высокую производительность, эффективность и нагрузоустойчивость информационных ресурсов предприятий и организаций. Фактически, решения и оборудование Cisco Systems создают надежный аппаратносетевой фундамент корпоративной сети для корпораций, госучреждений, университетов, правительственных организаций во всем мире.

Для небольших компаний, относящихся к сегменту SMB, Cisco выпустило ряд специальных решений, способных существенно увеличить производительность работы локальных сетей, а также обеспечить интеграцию ЛКС, физически созданных в офисном здании, с удаленными приложениями.

Для внедрения функции приоритезации предлагается выбрать маршрутизатор Cisco 2911, полностью отвечающий поставленной задаче [2]. Маршрутизатор обладает следующими характеристиками:

\section{Таблица 1 - Характеристики маршрутизатора Cisco 2911}

\begin{tabular}{|c|c|}
\hline \multicolumn{2}{|l|}{ Интерфейсные порты: } \\
\hline WAN/LAN-интерфейсы: & 3 порта Ethernet 10Base-T/100Base-TX/1000Base-T, разъем RJ-45; \\
\hline Другие интерфейсы: & $\begin{array}{l}1 \text { консольный порт управления, разъем RJ-45; } \\
1 \text { консольный порт управления, коннектор Mini-USB тип B; } \\
1 \text { последовательный вспомогательный порт, разъем RJ-45; } \\
2 \text { порта USB 4-пин USB тип A. }\end{array}$ \\
\hline Слоты расширения: & $\begin{array}{l}\text { 4/4 слота для HWIC; } \\
\text { 2/1 слота для карт CompactFlash; } \\
1 / 1 \text { слот расширения; } \\
1 / 1 \text { слот дпя SFP (или mini-GBIC). }\end{array}$ \\
\hline \multicolumn{2}{|l|}{ Сетевые особенности: } \\
\hline Производительность: & до $75 \mathrm{Mbps}$ \\
\hline LAN-сервисы: & IEEE 802.1Q, IEEE 802.1ag, IEEE 802.1ah, IEEE 802.3af, IEEE 802.3ah. \\
\hline Сетевая безопасность: & $\begin{array}{l}\text { Cisco Security Manager; VPN encryption; Cisco IOS Firewall; Cisco IOS Zone-Based } \\
\text { Firewall; Cisco IOS IPS; Cisco IOS Content Filtering; AAA; DES; 3DES; AES }\end{array}$ \\
\hline Протоколы маршрутизации: & $\begin{array}{l}\text { BGP, GRE, OSPF, DVMRP, EIGRP, IS-IS, IGMPV3, PIM-SM, PIM-SSM, статическая IPV4 и } \\
\text { IPv6 маршрутизация. }\end{array}$ \\
\hline QOS: & $\begin{array}{l}\text { поддерживает: VPN, DMVPN, IPv6, MPLS, Syslog; } \\
\text { установлены: фаервол, функция фильтрации контента, DMVPN, WRED, CBWFQ. }\end{array}$ \\
\hline Управление: & $\begin{array}{l}\text { CiscoWorks LAN Management Solution (LMS); CiscoWorks QoS Policy Manager (QPM); } \\
\text { Cisco Configuration Engine; SNMP; Syslog; * NetFlow; CLI }\end{array}$ \\
\hline \multicolumn{2}{|l|}{ Характериспики памяяг: } \\
\hline Флеш-память: & $\begin{array}{l}\text { - По умолчанию: } 256 \text { МБ } \\
\text { - Максимум: } 4 \text { ГБ } \\
\end{array}$ \\
\hline Оперативная память: & $\begin{array}{l}\text { - По умолчанию: } 512 \text { МБ } \\
\text { - Максимум: } 2 \text { ГБ } \\
\end{array}$ \\
\hline \multicolumn{2}{|l|}{ Физические характеристики: } \\
\hline Размеры, мм: & $88.9 \times 438.2 \times 469.9$ \\
\hline Вес, кг: & $\begin{array}{l}8.2 \text { кг (с источником питания, без модулей); } \\
8.6 \text { кг (с РоЕ, без модулей); } \\
9.5 \text { кг (типичный вес в полной конфигурации). } \\
\end{array}$ \\
\hline Параметры питания: & Внутренний блок питания. 120/230 В переменного тока. \\
\hline Форм-фактор: & Внешний. Занимает 2 юнита. \\
\hline
\end{tabular}




\section{6. Настройка}

В рамках решения задачи по внедрению функции приоритезации трафика в сеть предприятия, необходимо удовлетворить следующие требования по приоритезации:

- мультимедиа трафик критичен к задержкам и потерям, должен проходить между офисами компании с наивысшим приоритетом;

- на мультимедиа трафик должно быть гарантировано не менее $45 \%$ пропускной способности канала;

- терминальный трафик должен иметь льготные условия прохождения по сети;

- на терминальный трафик должно быть гарантировано не менее $20 \%$ пропускной способности канала.

Перейдем к рассмотрению детальной структуры командной строки управления QoS в устройствах Cisco. Данная модель является центральной в QoS решениях построенных на базе Cisco IOS. В оригинале она звучит как Modular QoS CLI, (MQC).

$\mathrm{MQC}$ разделяет задачи связанные с QoS на следующие модули [5, 6]:

- определение потока трафика;

- классификация его на принадлежность к классу QoS;

- применение политик QoS для данного класса;

- определение интерфейсов, на которых политика должна быть приведена в исполнение.

Внутри сети предприятия должны быть введены дополнительные классы трафика, $\mathrm{AF} 11$ и AF12, для маркировки исходящего от мультимедиа оборудования трафика.

Весь трафик, который содержит значения AF11 или AF12 в байте ToS, будет частью класса известного как RTP (Real-time Transport Protocol).

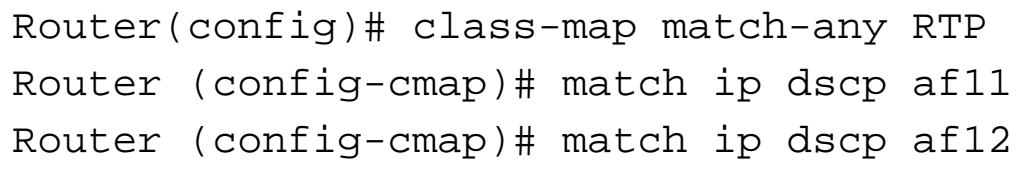

Команда class-map используется для того, чтобы описать класс трафика, Назначение класса трафика - классифицировать или идентифицировать трафик который будет передан конкретному QoS.

Команда match используется для определения различных критериев классификации пакетов. Если пакет совпадает с указанными критериями:

- пакет относится к данному классу;

- пакет пересылается, следуя спецификациям QoS, указанными в политике трафика.

\section{7. Описание класса для передачи терминального трафика}

Для этого потребуется создать пакетный фильтр, разрешающий передачу трафика на определенные порты, использующиеся для терминальных соединений. 
Пакетные фильтры позволяют управлять прохождением трафика через интерфейсы роутера, разрешая или запрещая передачу пакетов, удовлетворяющих указанным условиям. Пакетные фильтры используются в качестве базового средства обеспечения безопасности сети.

Пакетные фильтры в Cisco реализованы через списки доступа ACL (Access Control List).

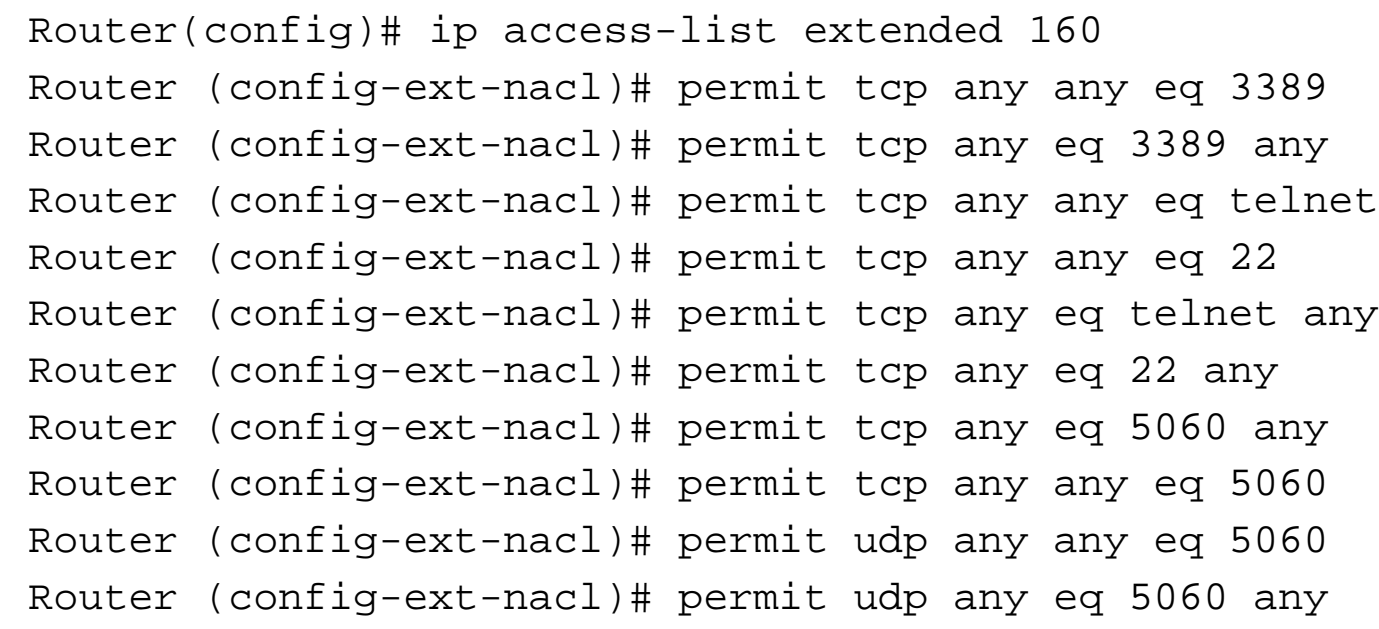

Следующим шагом описываем сам класс трафика:

Router(config)\# class-map match-any RDP

Router (config-cmap)\# match access-group 160.

Весь трафик, который разрешен через ACL 160, будет частью класса известного как RDP (протокол удалённого рабочего стола).

\section{8. Политика для трафика}

Команда policy-map используется для создания и назначения политики трафика, то есть конфигурирование функций QoS, которые должны быть связаны с трафиком, классифицированным как трафик, описанный пользователем. Политика трафика состоит из трех элементов:

- $\quad$ имя политики (Policy name);

- $\quad$ класс трафика (обозначается командой class);

- политики QoS, которые будут применены к каждому классу.

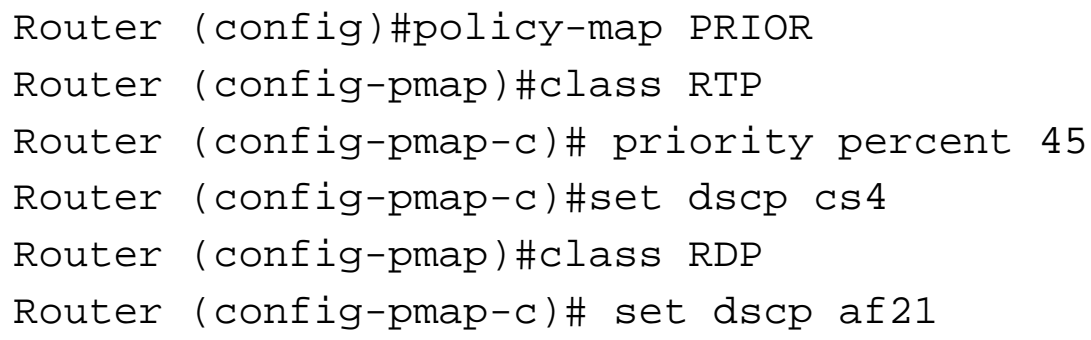


Router (config-pmap-c) \#priority percent 20

Router (config-pmap) \#class class-default

Данный policy-map создает политику трафика, называющуюся PRIOR. Политика применяется ко всему трафику, классифицируемому заранее описанными классами трафика «RTP» и «RDP». Указывает, что трафик RTP следует промаркировать как CS4 и предоставить полосу пропускания 45\%. Трафик RDP следует промаркировать как af21 и предоставить полосу пропускания $20 \%$.

Весь трафик, который не принадлежит данным классам, формирует часть класса class-default.

\section{9. Добавление политики трафика к интерфейсу}

Команда service-policy используется для присоединения политики трафика, указанной командой policy-map, на интерфейс.

Может быть применена как для входящих, так и для исходящих пакетов на указанном интерфейсе, поэтому в данной команде необходимо указывать направление трафика.

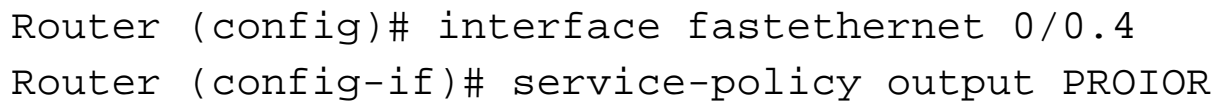

Bce пакеты, покидающие указанные интерфейс, должны быть совместимы с критериями, указанными в политике трафика, названной PROIOR.

\section{0. Заключение}

Существуют различные типы трафика, отличающиеся чувствительностью к задержкам потерям пакетов. Наиболее грубая классификация трафика разделяет его на два класса: трафик реального времени (чувствительный к задержкам) и эластичный трафик (нечувствительный к задержкам в широких пределах). Методы QoS основаны на перераспределении имеющейся пропускной способности линий связи между трафиком различного типа в соответствии с требованиями приложений. В этом случае можно решить проблему передачи мультимедийного трафика, повысив приоритет передаваемой информации. Для внедрения функции приоритезации подходит оборудование Cisco. В статье показан пример настройки выбранного маршрутизатора, полностью отвечающий поставленной задаче.

\section{Список информационных источников}

[1] Таненбаум, Э.С. Компьютерные сети. 5-е изд. / СПб: Питер, 2012. - с. 762.

[2] Филимонов, А.Ю. Построение мультисервисных сетей Ethernet / А.Ю. Филимонов. - СПб.: БХВ-Петербург, 2007. - 502 с. 
[3] Блинова В.М. Метод контроля функционирования передачи данных в автоматизированной системе управления промышленным предприятием // Промышленные АСУ и контроллеры. - 2012. - №3. - С.34-38.

[4] Пескова, С. А. Сети и телекоммуникации: учеб. пособие для вузов / С.А. Пескова, А.В. Кузин, А.Н. Волков. - 2-е изд., стер. - М.: Академия, 2007. - 350 с.

[5] Network.xsp [http://network.xsp.ru.].

[6] Products_ios_technology_home[http://www.cisco.com/en/US/products].

[7] Антонов П.Д. User Is A Great Obstacle For Security Systems / П.Д. Антонов, А.В. Остроух // Молодой ученый. - 2011. - №4. Т.3. - С. 62-63.

[8] Исмоилов М.И. Подготовка и переподготовка персонала предприятий промышленного и транспортного комплексов с применением мобильных технологий: монография / М.И. Исмоилов, А.Б. Николаев, А.В. Остроух. - SaintLouis, MO, USA: Publishing House Science and Innovation Center, 2013. - 166 c. ISBN 978-0-615-67111-6.

[9] Николаев А.Б. Информационные технологии в менеджменте и транспортной логистике: учебное пособие / А.Б. Николаев, А.В. Остроух. - Saint-Louis, MO, USA: Publishing House Science and Innovation Center, 2013. - 254 c. - ISBN 978-0615-67110-9.

[10] Остроух А.В. Информационные технологии в научной и производственной деятельности / [ред. А.В. Остроух] - М: ООО "Техполиграфцентр", 2011. - 240 с. ISBN 978-5-94385-056-1.

[11] Остроух А.В. Ввод и обработка цифровой информации: учебник для нач. проф. образования / А.В. Остроух. - М.: Издательский центр «Академия», 2012. - 288 с. - ISBN 978-5-7695-9457-1.

[12] Белоусова А.И. Подход к формированию многоуровневой модели мультиагентной системы с использованием миваров / А.И. Белоусова, О.О. Варламов, М.Н. Краснянский, А.В. Остроух // Перспективы науки - Тамбов. «ТМБПринт», 2011. № 5(20). - С. 57-61.

[13] Остроух А.В. Системы искусственного интеллекта в промышленности, робототехнике и транспортном комплексе: монография / A.В. Остроух Красноярск: Научно-инновационный центр, 2013. - 326 с. - ISBN 978-5-906314$10-9$.

[14] Остроух А.В. Автоматизация и моделирование работы предприятий по строительству промышленных объектов: дис. ... д-ра техн. наук: 05.13.06: защищена 07.04.09: утв. 19.06.09. - М., 2009. - 357 с.

[15] Остроух А.В. Автоматизация и моделирование работы предприятий по строительству промышленных объектов: автореф. дис. ... д-ра техн. наук: 05.13.06. - М., 2009. - 43 c. 